\title{
Il pharmakos nelle fonti antiche e nella Storia delle religioni. Alcune valutazioni critiche
}

The pharmakos in the Earliest Sources and in History of Religions. Some Critical Evaluations

Leonardo Sacco

\section{OpenEdition}

\section{Journals}

\section{Edizione digitale}

URL: https://journals.openedition.org/mythos/325

DOI: $10.4000 /$ mythos.325

ISSN: 2037-7746

\section{Editore}

Salvatore Sciascia Editore

\section{Edizione cartacea}

Data di pubblicazione: 1 décembre 2018

Paginazione: 103-115

ISBN: 978-88-8241-501-3

ISSN: $1972-2516$

\section{Notizia bibliografica digitale}

Leonardo Sacco, «ll pharmakos nelle fonti antiche e nella Storia delle religioni. Alcune valutazioni

critiche», Mythos [Online], 12 | 2018, online dal 24 septembre 2019, consultato il 21 septembre 2021

URL: http://journals.openedition.org/mythos/325 ; DOI: https://doi.org/10.4000/mythos.325 


\section{II pharmakos nelle fonti antiche e nella Storia delle religioni. Alcune valutazioni critiche}

\section{Leonardo Sacco}

\section{Riassunto}

Cosa s'intende con il termine greco pharmakos? Si trattava di un rito di purificazione oppure di un sacrificio? Quali sono le origini di questa cerimonia? Uno o due individui - in caso di crisi sociale, religiosa, politica, o a fronte di una calamità naturale - erano espulsi dalla comunità (e, talvolta, uccisi ritualmente) poiché si riteneva che tale pratica avrebbe purificato la società. Alcune fonti sembrano asserire che il pharmakos fosse sacrificato, ma la dottrina prevalente rigetta questa tesi adducendo che la vittima era percossa, ma non uccisa. Una spiegazione più plausibile vorrebbe che in talune occasioni fosse giustiziato, mentre in altre no. In questa prospettiva, esiste lo spazio per una problematizzazione storico-religiosa?

\begin{abstract}
What is meant by the Greek word pharmakos? Was it a purification ritual or a sacrifice? What are the origins of this ceremony? One or two individuals - in case of social, religious, political crisis, or a natural disaster - were expelled from the community (and sometimes ritually killed) because it was believed that this would bring about the purification of the human group. Some sources state that the pharmakos was sacrificed, but several scholars oppose such a thesis, arguing that the earliest sources for the ritual prove that the victim was beaten up, but not killed. A more plausible explanation would be that on certain occasions he was executed, while on others he was not. In this light, is there a space for a historical-religious problematization?
\end{abstract}

\section{Parole chiave}

Pharmakos - rito - sacrificio - capro espiatorio • ostracismo • devotion Storia delle religioni (metodo comparativo)

\section{Keywords}

Pharmakos - ritual - sacrifice - scapegoat - ostracism • devotion History of Religions (Comparativism)

\footnotetext{
Oosa s'intende con il termine greco pharmakos? Si trattava di un rito di purificazione o di un sacrificio? Quali sono le sue origini? Esiste lo spazio per una problematizzazione storico-religiosa?
}

Dall'analisi integrata dei passi dello scrittore ellenistico Istro (III secolo a.C.) e del grammatico di Antinoupoli sul Nilo Ellàdio (IV secolo d.C.) apprendiamo che ad Atene erano soliti espellere una coppia di persone affinché la città fosse purificata durante le feste di Thargelia ${ }^{1}$. Secondo il racconto, i due individui, detti pharmakoi (secondo Istro) o Sybakchoi (secondo Ellàdio), uno in rappresentanza degli uomini, adornato con fichi neri intorno al collo, e uno in rappresentanza delle donne, adornato con fichi bianchi, venivano scacciati dai confini della polis. La purificazione serviva a scongiurare le conseguenze malevole di una disgrazia che, per

1 Durante le feste Thargelia, solennità celebrate ad Atene e in altri centri ionici, in onore di Apollo, il 6 e il 7 del mese Thargelion (maggio), avevano luogo una purificazione, un sacrificio a Demetra e una processione. Il rito purificatorio consisteva nella cacciata dalla polis di due uomini (o di un uomo e una donna), in qualità di emissari, scelti fra le persone più ripugnanti (talvolta fra i criminali), e aveva la funzione di stornare tutte le impurità dalla polis. Per una descrizione esaustiva, cfr. Gebhard 1926, 68-88; Pestalozza 1930-31; Nilsson 1950, I, 97-100. 
la prima volta, ebbe luogo con la morte prematura e ingiusta di Androgeo il cretese (figlio del re Minosse) e che, da quel momento, continuò ad essere in vigore ${ }^{2}$.

Tale fattispecie originava dall'idea che si potesse trasferire il male (fisico e/o morale) da un oggetto (e/o da un essere animato) sopra un altro oggetto (e/o un altro essere animato) che, poi, veniva allontanato (e/o ucciso) ${ }^{3}$. Paradigmatico era, in Israele, il caso del "capro espiatorio", sul quale il sommo sacerdote, vestito di lino, e dopo le opportune lustrazioni, accumulava tutte le colpe e i peccati del popolo, imponendogli ambo le mani sul capo; l'animale veniva poi condotto fuori dalla comunità da un uomo che avrebbe dovuto lasciarlo in un luogo deserto ${ }^{4}$.

In alcuni frammenti dei giambi del poeta Ipponatte, raccolti dallo studioso bizantino Tzetzès, emerge che la cerimonia - nelle città ioniche - si svolgeva durante le celebrazioni di Thargelia 5 . $\mathrm{Al}$ rito propiziatorio per un buon raccolto, Tzetzès associa pure circostanze straordinarie: se la disgrazia o la carestia, o la peste, o un'altra malattia si abbattevano sulla città per la collera divina, l'uomo più abietto di tutti era condotto al sacrificio per mondare e curare la comunità sofferente ${ }^{6}$. A Massalia, fondata dai Focesi nel VI secolo a.C., il rito è ancora noto nel I secolo d.C., essendo menzionato in un frammento del Satyricon di Petronio, fonte comune, secondo Hughes, di Servio e di Stazio: i pharmakoi, in questo caso, dopo essere stati nutriti a spese della popolazione, venivano espulsi in un dato giorno e uccisi ${ }^{7}$. Nella località di Abdera (in Tracia), un uomo, dopo essere stato tenuto in isolamento, veniva fatto uscire dai confini e lapidato, per

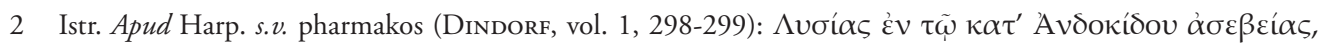

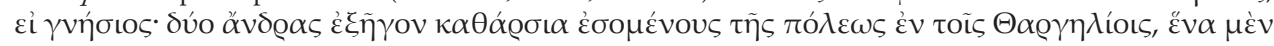

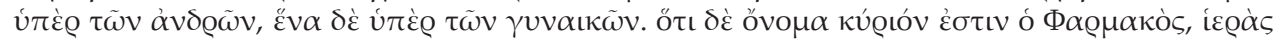

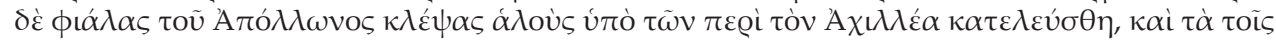

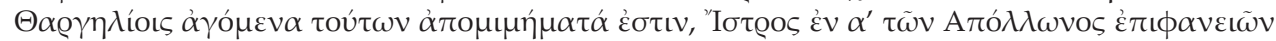

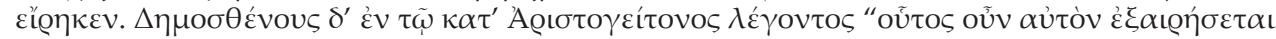

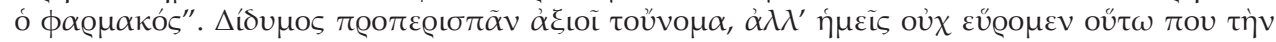

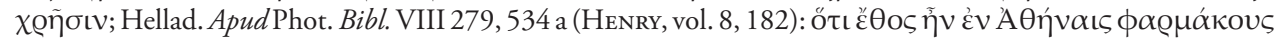

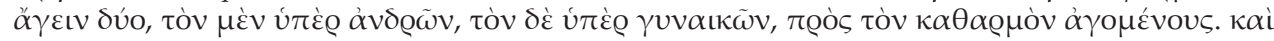

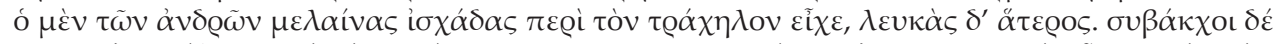

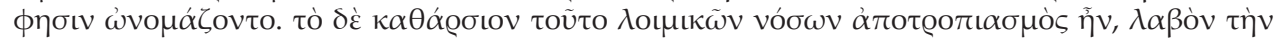

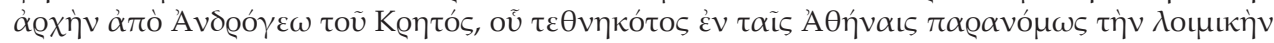

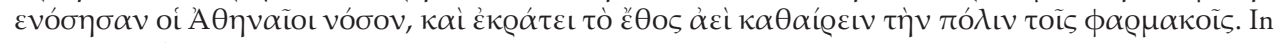
letteratura, cfr. Deubner 1932, 179-198, praes. 179-181; Bodnár 2000, 750.

3 In quest'ottica, MCLEAN 1990 ha proposto di non rendere il greco pharmakos con il tradizionale termine scapegoat, bensì con scapeman, la cui rimozione (espulsione) permetteva di raggiungere il fine prefissato della purificazione.

4 Levit. XVI 21-22: Aronne poserà le mani sul capo del capro vivo, confesserà sopra di esso tutte le iniquità degli Israeliti, tutte le loro trasgressioni, tutti i loro peccati e li riverserà sulla testa del capro; poi, per mano di un uomo incaricato di ciò, lo manderà via nel deserto. Quel capro, portandosi addosso tutte le loro iniquità in una regione solitaria, sarà lasciato andare nel deserto. Nel merito, cfr. FeInberg 1958; Davies 1977; Zatelli 1998; Westbrook, LeWIs 2008.

5 Masson 1962, fr. 104. Supra, n. 1.

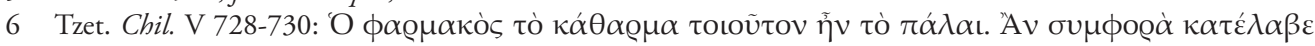

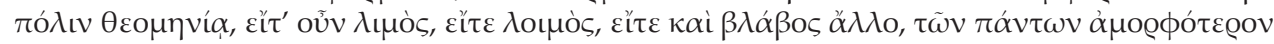

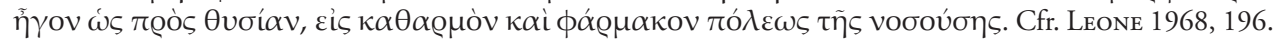

7 Petr. fr. 1 = Serv. Ad Aen. III 57: Nam Massilienses quotiens pestilentia laborabant, unus se ex pauperibus offerebat alendus anno integro publicis <sumptibus > et purioribus cibis. Hic postea ornatus verbenis et vestibus sacris circumducebatur per totam civitatem cum execrationibus, ut in ipsum reciderent mala totius civitatis, et sic proiciebatur. Cfr. Hughes 1991, 146. 
espiare le perversioni dei suoi concittadini ${ }^{8}$. Il pharmakos, dunque, non sarebbe stato solamente un rito propiziatorio o di espiazione/purificazione, ma anche un sacrificio, sebbene per l'età classica paia sicuro come la pratica rituale non fosse più cruenta". Il "sacrificio" costituirebbe un "caso limite", poiché l'uccisione rituale della vittima (che seguiva la sua cacciata) si verificava senza alcun riferimento a un destinatario: un fatto che, teoricamente, non aveva carattere di sacrificio ${ }^{10} \mathrm{ma}$, essendo incluso in un determinato culto divino, poteva essere concepito come tale ${ }^{11}$.

Le prime attestazioni di un rito di espulsione/purificazione del tipo di quello greco del pharmakos si trovano in alcune tavolette cuneiformi dalle quali sembrerebbe emergere che la cerimonia fosse già eseguita a Ebla oltre cinquemila anni fa ${ }^{12}$. Nei testi hittiti, poi, si possono reperire circostanze paragonabili: qui si fa affidamento sull'espulsione coatta - ad opera del re e dei suoi ufficiali più alti in grado - di due montoni decorati e una donna finemente vestita per allontanare la peste dalla città, guarendo simultaneamente il popolo e l'esercito ${ }^{13}$.

In una prospettiva metodologica d'impronta storico-religiosa, e sulla scorta di talune as-

8 Schol. Ov. Ibis 467-468 (Elus 1881, 81): Mos erat in Abdera civitate singulis annis hominem inmolari pro peccatis ciuium, sed prius vii diebus excommunicari ut sic omnium peccata solus haberet. Callimachus dicit quod Abdera est civitas in qua talis est mos, quod uno quoque anno totam ciuitatem publice lustrabant, et aliquem ciuium quem in illa die habebant deuotum pro capitibus omnium lapidibus occidebant. Cfr. Deubner 1934; Gras 1984, 78-82; Brunaux 1988, 131-132; Hughes 1991, 157.

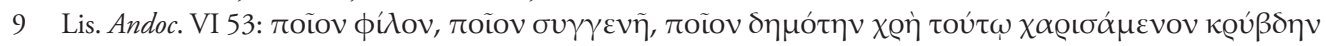

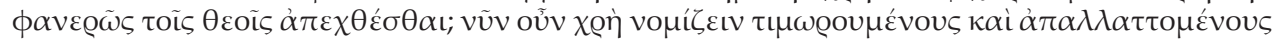

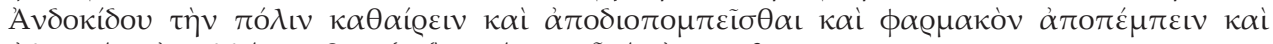

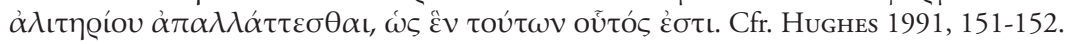

10 Harrison 1903, 95-114; Farnell 1907, IV, 268-284; 253-258; Frazer 191333, 252-274. Il sacrificio - com’è noto - è un atto di culto rituale contemplato in quasi tutte le tradizioni religiose, che implica una peculiare sottomissione al sacro e la volontà di stabilire una relazione con esso. Cfr. Yerkes 1952, 3-6. L'espressione origina dal latino sacrificium (sacrum + facere) che, etimologicamente, fa riferimento all'azione di "rendere sacro". Tutti i generi di sacrificio hanno un denominatore comune, ovvero il presupposto che vi siano: un sacrificante (persona o gruppo), un oggetto (offerta) o un essere (vittima animale o umana) da sacrificare, un rito di trasferimento dell'oggetto/essere - mediante distruzione, consumazione, abbandono - e, pertanto, un passaggio dalla sfera profana alla sfera sacra. Cfr. Brelich 1966, 44-50; VAN BAAL 1976, 161-178. Sulle principali teorie relative al sacrificio, cfr. Tylor 1871; Hubert, Mauss 1899; Durkheim 1912; Loisy 1920; Girard 1972; Burkert 1972; Detienne 1979; Grottanelli-Parise 1988; Carter 2003; Hedley 2011; Lawtoo 2017, 119-126.

11 BiANCHI 1989, 165, n. 1, in merito, notò che «...gli altri sacrifici umani regolari erano per i Greci quelli collegati con Zeus Laphystios, quelli del pharmakos e, forse, i Kronia di Rodi (Porph. Abst. II 54)».

12 Xella 1996; Zatelli 1998.

13 Gurney 1977, 49: «When evening comes, whoever the army commanders are, each of them prepares a ram whether it is a white ram or a black ram does not matter at all. Then I twine a chord of white wool, red wool, and green wool, and the officer twists it together, and I bring a necklace, a ring, and a chalcedony stone and I hang them on the ram's neck and horns, and at night they tie them in front of the tents and say: "Whatever deity is prowling about (?), whatever deity has caused this pestilence, now I have tied up these rams for you, be appeased!" And in the morning I drive them out the plain, and with each ram they take I jug of beer, I loaf, and I cup of milk (?). Then in front of the king's tent he makes a finely dressed woman sit and puts with her a jar of beer and three loaves. Then the officers lay their hands on the rams and say: "Whatever deity has caused this pestilence, now see! These rams are standing here and they are very fat in liver, heart, and loins. Let human flesh be hateful to him, let him be appeased by the rams". And the officers point at the rams and the king points at the decorated woman, and the rams and the woman carry the loaves and the beer through the army and they chase them out of the plain. And they go running on the enemy's frontier without coming to any place of ours, and the people say: "Look! Whatever illness there was among men, oxen, sheep, horses, mules, and donkeys in this camp, these rams and this woman have carried it away from the camp". And the country that finds them shall take over this evil pestilence». 
serzioni suffragate da studi eminenti, si tenterà di fornire una serie di valutazioni critiche a proposito del pharmakos e di altre tipologie rituali e votive, quali "ostracismo", "capro espiatorio", "devotio", che presentano, tra loro, affinità e differenze, con l'obiettivo di pervenire a un quadro più articolato della questione ${ }^{14}$, provando a "riaprire" - forse - le sorti di un dibattito formalmente chiuso giacché nella storiografia moderna - come è noto - prevale la tesi secondo la quale il pharmakos non debba essere annoverato fra i "sacrifici"15.

Walter Burkert (1931-2015) - basandosi su Tzet. Chil. V 728-739 - ha mostrato come il pharmakos fosse espulso e sacrificato (per olocausto), dopo essere stato ben curato e sfamato dalla città, e come le sue ceneri fossero sparse nel mare ${ }^{16}$ : si trattava di un sacrificio cruento, ma pure di una forma di catarsi sociale ${ }^{17}$. La funzione del pharmakos era quella di sanare apotropaicamente la polis, incarnandone le negatività e portandole fuori da essa, assumendo le vesti simboliche di un bouc émissaire ${ }^{18}$. Occorre, però, domandarsi se questi venisse espulso in quanto personificazione della sventura collettiva, o come inviato presso gli dèi. La differenza sembrerebbe marginale, nondimeno proprio in tale ottica la comparazione aiuta a definire meglio i contorni della fattispecie poiché Erodoto, nel tracciare le specificità del sacrificio umano officiato dai Geti, illustra una particolarità consona al pharmakos, inteso come espulsione di un individuo. Presso questa popolazione, infatti, la vittima oggetto del rito era considerata latrice di un messaggio per le divinità ${ }^{19}$, un aspetto questo che - benché scientificamente debole e isolato $^{20}$ - potrebbe indurre a ritenere come anche il pharmakos non rivelasse un rituale esclusivamente purificatorio.

14 Il richiamo al "comparativismo storico" implicherebbe - per la rilevanza del tema - uno studio più approfondito che, però, in questa sede, non appare possibile. Tuttavia, non si possono tralasciare alcune note sintetiche quanto indispensabili per la sua comprensione e per la redazione del presente studio. La Storia delle religioni, permettendo di accostare ciò che è noto a ciò che non lo è, costituisce la mediazione tra la somiglianza dei concetti e la difformità dei dati: tale metodo, pertanto, confrontando elementi d'ambito differente, e sottoponendo le argomentazioni derivanti dalla contrapposizione ad un'analisi imparziale, mira a scoprire analogie oggettive, ma soprattutto diversità sul piano culturale. Per una bibliografia di riferimento, cfr. Pettazzoni 1924; DE Martino 1953-54; Brelich 1956; Pettazzoni 1959; Bianchi 1970; Brelich 1970-72; Brelich 1977; Sabbatucci 1978; Gasbarro 1990; Pisi 1990; Filoramo 1997; Montanari 2001; Casadio 2011; Sacco 2016.

15 Hughes 1991, 140-143.

16 Burkert 1985, 82; in letteratura: Bremmer 1983, 300; Paoletti 2004, 33-35. Contrari alla tesi del "sacrificio cruento", per esempio: NAGY 1979, 222-242; Hughes 1991, 139-165, secondo il quale, nella maggior parte dei casi esaminati (ma non nella totalità di essi-eccezione nostra), la morte del pharmakòs non appare evidente; di più ampio respiro GreEN 1998.

17 BONNECHERE 1994.

18 Girard 1982.

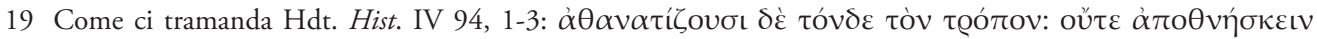

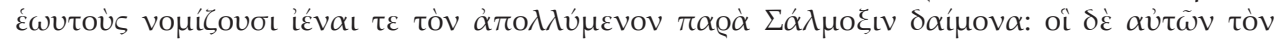

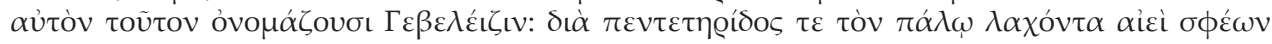

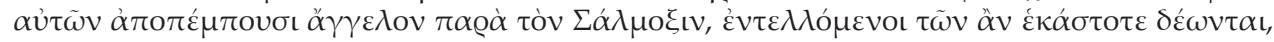

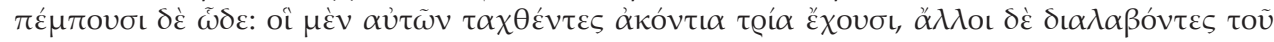

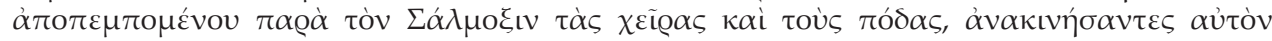

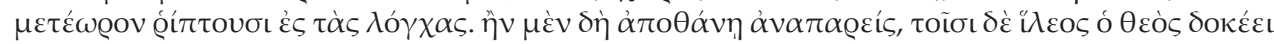

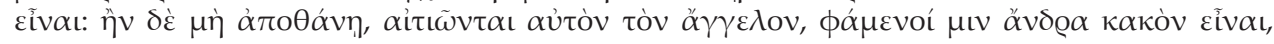

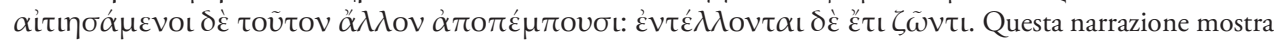
analogie morfologiche tanto con la devotio quanto con il pharmakos e ricorda, per certi versi, l'ordalia (Lat. iudicium dei), per la quale v. Glotz 1904. Per un approccio complessivo, cfr. STERn 1991.

20 Lawson 1910, 356. 
Jean-Pierre Vernant (1914-2007) ha notato un'analogia funzionale tra capro espiatorio e ostracismo. Nella persona dell'ostracizzato la città rimuoveva ciò che era in essa troppo elevato e incarnava il male che poteva venirle dall'alto. Nella persona del pharmakos, viceversa, la città ripudiava ciò che essa riteneva di più abietto e impersonava il male che la minacciava dal basso. Con tale rigetto, doppio e complementare, la polis si delimitava in relazione a un aldilà e a un aldiquà: prendeva la misura propria dell'umano in contrapposizione, da un lato al sacro e all'eroico, dall'altro al bestiale e al mostruoso ${ }^{21}$. L'ostracismo era ritenuto uno strumento con cui si sarebbe dovuta evitare la formazione di una tirannide ${ }^{22}$ : tesi basata su un passo della Politica di Aristotele ${ }^{23}$, e sulla circostanza che il primo ateniese ostracizzato, Ipparco, appartenesse alla famiglia dei Pisistratidi; ipotesi confermata dal fatto che anche successivamente sarebbero state ostracizzate persone provenienti dall'ambiente dei primi tiranni. Tali argomenti, però, non sembrano del tutto convincenti e le antiche testimonianze sulla funzione dell'ostracismo appaiono contraddittorie ${ }^{24}$. Inoltre, un'interpretazione fondata unicamente su Aristotele non pare scientificamente ammissibile, perché non si avvale delle fonti del V secolo ${ }^{25}$.

Hendrik Versnel (1936-), dal canto suo, ha trovato qualche affinità tra devotio e pharmakos, arrivando a definire Decio Mure a prototypical pharmakos ${ }^{26}$. Nella devotio un magistratus provvisto di imperium militiae (consul, dux, praetor) - o un "privato cittadino" (civis designatus) scelto tra i legionari dell'exercitus sul campo di battaglia - dopo essersi votato alle divinità infere e alla Terra, osservando un rito codificato dalla "teologia pontificale" 27 , si lanciava tra le schiere nemiche, con lo scopo di trovarvi la morte, per garantire la vittoria alla propria parte e salvare l'integrità della res publica ${ }^{28}$. In questa chiave, il Burkert ha discusso la devotio alla luce della pharmakos ideology $y^{29}$ : l'individuo espulso dalla comunità raffigurava, insieme, "delitto" e "salvatore", che mediante il proprio sacrificio permetteva alla società di ritrovare sicurezza, assicurandole prosperità.

In virtù di quanto esposto paiono essenziali alcune considerazioni.

L'etnologo Arnold van Gennep (1873-1957) ha osservato che: "per sopravvivere ogni società deve soddisfare due requisiti fondamentali: la coesione interna e la continuità temporale» ${ }^{30}$. Perciò, quel che ha consentito alle istituzioni arcaiche di superare le crisi è stata la risoluzio-

21 Vernant, Vidal-Naquet 1977, 97. Sull'interpretazione del concetto di “mostruoso" nell'antica Grecia e, più in generale, nell'Antichità classica, cfr. BAgLioni 2013, 15-32.

22 CARCOPINO 1935, 96-97.

23 Arist. Pol. III 1284 a. Cfr. Reeve 1998, lxix-lxxii; 89-91.

24 Kagan 1961; BucKley 2010², 132-136.

25 Cfr. Burns 1981; Missiou 2011, 36-149; Stoneman 2013.

26 Versnel 1981, 139 (per la citazione); v. anche: Versnel 1977, 37-46.

27 Liv. VIII 9, 6-8: Iane, Iuppiter, Mars pater, Quirine, Bellona, Lares, Diui Nouensiles, Di Indigetes, Diui, quorum est potestas nostrorum hostiumque, Dique Manes, uos precor ueneror, ueniam peto feroque, uti populo Romano Quiritium uim uictoriam prosperetis hostesque populi Romani Quiritium terrore formidine morteque adficiatis. sicut uerbis nuncupaui, ita pro re publica [pouli Romani] Quiritium, exercitu, legionibus, auxiliis populi Romani Quiritium, legiones auxiliaque hostium mecum Deis Manibus Tellurique deuoueo.

28 La tradizione attribuisce questo gesto a tre componenti della gens Decia: Publius Decius Mus al Veseris (340 a.C.), suo figlio a Sentinum (295 a.C.), e suo nipote ad Ausculum (279 a.C.), sebbene, in letteratura, la storicità delle tre vicende non sia pacifica e solo della seconda si disponga di un'apprezzabile documentazione. Cfr. SACCO 2011, 83-93.

29 BURKERT 1979, 59-77.

30 VAN GenneP 1981, 15. 
ne violenta, ma purificatrice, del "tutti contro uno"31. Dopo l'espulsione (sacrificio) unanime di colui che veniva identificato come il "colpevole della crisi" (in senso figurato "il capro espiatorio"), la comunità ritrovava "magicamente" l'armonia ${ }^{32}$. L'identificazione nel pharmakos dell'essere mostruoso, lasciato ai margini, costituisce la preparazione necessaria alla sua immolazione. Nelle culture antiche, d'altronde, l'“ultimo" (che aveva sempre un aspetto ripugnante) era accolto e curato, ma nell'immaginario collettivo restava sempre la "vittima predestinata", proprio come accadeva al pharmakos ${ }^{33}$.

Secondo una fonte, il pharmakos, a Massalia, si sarebbe offerto spontaneamente alla morte $^{34}$, una prerogativa che paleserebbe una possibile volontarietà dell'atto ${ }^{35}$, ma secondo altre fonti l'“autosacrificio" sarebbe stato mosso dall'eventualità di ottenere una ricompensa, come peraltro sembrerebbe essersi verificato in Abdera $^{36}$. Fino al 1933 si è ritenuto che il pharmakos di Abdera fosse stato lapidato a morte, ma l'anno successivo fu rinvenuto un papiro (Diegeseis II 29-40) recante un frammento che stabiliva inequivocabilmente come la vittima fosse stata allontanata, ma non uccisa ${ }^{37}$; a Massalia, invece, sappiamo che il pharmakos veniva certamente sacrificato $^{38}$. Il Nilsson - per superare tali ostacoli ermeneutici - adottò un escamotage, considerando indifferente la sorte della vittima, essendo comunque raggiunto il fine del rito, ossia l'espulsione, anticipando - ci sembra - la riflessione di McLean, a proposito del valore semantico del termine scapeman in luogo di scapegoat ${ }^{39}$.

In merito all'ostracismo, va detto come questo non potesse rappresentare uno strumento adeguato contro la tirannide: se si considera, infatti, che esso veniva compiuto, al massimo, una volta all'anno e che la nascita di una tirannide costituiva un evento cronologicamente e storicamente non prevedibile, non si spiega come si sarebbe potuto impedire il suo avvento ricorrendo all'istituto ${ }^{40}$. Peraltro, la tesi formulata da Sara Forsdyke (1967-), secondo la quale l'ostracismo rappresenterebbe un rituale collettivo simbolico conforme a quello del capro espiatorio ${ }^{41}$, non è del tutto plausibile, in quanto tale interpretazione, ispirata forse dalla

31 Palaver 2013, chap. 4.

32 Girard 1980, 22.

33 Bremmer 2001, 174-210; Kearney 2003.

34 Petr. fr. 1 (MCLean 1996, 97).

35 Roussel 1922; Meuli 1975, 993-996. Infra - nel testo e in nota (44) - con riferimento alla devotio.

36 Stat. Theb. X 793-794: lustrare ciuitatem humana hostia Gallicus mos est. nam aliquis de egentissimis proliciebatur praemiis ut se ad hoc uenderet. qui anno toto publicis sumptibus alebatur purioribus cibis, denique certo et sollemni die per totam ciuitatem ductus ex urbe extra pomeria saxis occidebatur a populo. Cfr. Hughes 1991, 158.

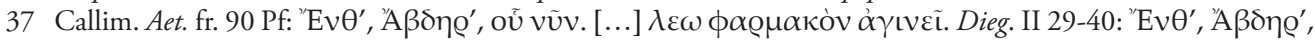

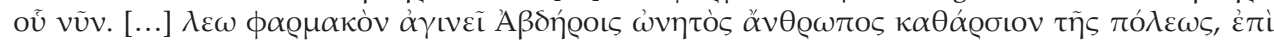

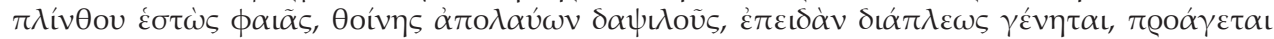

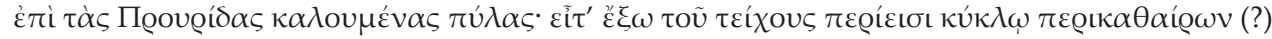

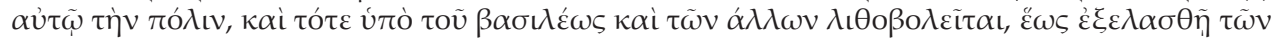
ógí $\omega v$. Cfr. Pfeiffer 1934.

38 Granite 2016, 216: «At Massalia (modern Marseille, France), the pharmakòs [...] was cast into the sea, purifying the city and its inhabitants».

39 Nilsson 1950, I, 97-100; McLean 1990; supra, n. 3.

40 FORSDYKe 2005, 144-177.

41 FoRSDYKE 2005, 145: «I argue that consideration of the procedure of ostracism as a form of collective ritual helps to explain the myriad associations and explanations for ostracism in the ancient sources»; 157: «Related to this interpretation of ostracism is the idea that the procedure may be associated with scapegoating and other types of rituals for expelling pollution». Cfr anche: OGDEN 1997, 15-28. 
"Scuola strutturalista francese" ${ }^{42}$, trascura una differenza rilevante: il fatto, cioè, che l'ostracismo non fosse un' espulsione simbolica e che, nelle fonti, la purificazione della polis da eventuali "macchie" non appare fondata su alcuna testimonianza ${ }^{43}$; elementi, questi, che distinguono nettamente l'ostracismo tanto dal rituale eponimo del capro espiatorio, quanto dall'espulsione del pharmakos.

Per quanto riguarda le riflessioni del Versnel, una giustapposizione fra devotus e pharmakos non appare condivisibile per almeno tre ragioni:

a) l'elemento dirimente tra le due pratiche riguarda, innanzitutto, il contesto di esecuzione: un momento disperato della battaglia per la devotio, una carestia o pestilenza collettiva per il pharmakos;

b) il devotus era un magistratus dotato di imperium militiae (o un civis designatus sul campo di battaglia) e, quindi, un "militare", laddove, invece, il pharmakos era un semplice cittadino un "civile", come diremmo oggi;

c) mentre il condottiero romano indossava un abito rituale e, prima di gettarsi fra i nemici, recitava una precisa formula, dettata dal pontifex maximus, il pharmakos, vestito in modo ridicolo e grottesco era scacciato dalla città, venendo a mancare - tra l'altro - il carattere della volontarietà dell'atto per motivi civici (la salvezza dello Stato), che costituisce l'elemento essenziale della devotio ${ }^{44}$. Il rito militare romano aveva un duplice obiettivo: assicurava la vittoria all'exercitus e conseguiva un obiettivo più elevato, la tutela e l'integrità della res publica ${ }^{45}$. Le perplessità riguardano, invece, la configurazione semantica del rito. Si trattava di un sacrificium o di un votum, o di qualcosa paragonabile ad entrambi? Tecnicamente, infatti, la devotio, più che al votum, somiglia alla preghiera seguita dal sacrificium e pare fondarsi sul pactum fra l'uomo e la divinità, volto a mantenere la pax deorum. Perciò, Decio Mure si configura come il paradigma dell'unus pro omnibus ${ }^{46}$ e di valori quali: pietas, ius, religio e virtus, manifestati fino all'autosacrificio - dando la percezione di trovarsi innanzi a un "sacrificio umano", o meglio a un "suicidio ritualizzato" 47 .

Sovente l'esplorazione specialistica tende a frazionare le analisi per singoli distretti e specifici indirizzi epistemologici. In tale sede, invero, l'ampliamento dei settori d'indagine, nonché l'utilizzazione di un criterio di ricerca esteso rispetto a quello tradizionale degli studi di storia antica, ha - forse - contribuito ad acquisire elementi e confronti la cui utilità, ai fini dello studio sul problema, non era stata finora ravvisata ${ }^{48}$. Più esattamente, il metodo comparativo ha consentito di elaborare una visione organizzata del tema. Partendo da talune antiche testimo-

42 Champagne 1992, 63-65; sul rapporto, a volte conflittuale, fra cultura, simbologia e strutturalismo, cfr. LEACH 1976; BotTuri 1983.

43 Secondo Kosmin 2015, si tratterebbe, invece, di un rituale politico atto a ristabilire la coesione interna della città.

44 Supra, n. 35.

45 Pettazzoni 1952, 18.

46 L'espressione è usata in Cic. Sest. XX 46.

47 Cfr. Brelich 1967; Durkheim 1897. Le percezioni, tuttavia, a volte sono fonte di perplessità. De facto, il devotus apparteneva agli dèi inferi e alla Terra e attirava il nemico nella propria consecratio; inoltre, qualora non fosse deceduto era previsto un rito di inumazione di un signum in sua vece, come attesta Liv. VIII 10, 12: Si is homo qui devotus est moritur, probe factum videri; ni moritur, tum signum septem pedes altum aut maius in terram defodi et piaculum caedi. Un'immagine - questa - che, in ambito storico-archeologico, ha suscitato un vasto dibattito, come testimoniano: Adams Holland 1956; Bö̈thius 1956; Berggren 1990; Ghenghea 2012.

48 Supra, n. 15. 
nianze, a proposito del rito oggetto d'indagine, si è preso atto che sovente l'analogia strutturale dell'impianto rituale non è sinonimo di analogia semasiologica, così - ad esempio - l'allontanamento di un animale poteva implicare la rimozione della peste (Ittiti), come pure dei peccati (Israeliti) da una comunità. Il rito, nelle sue diverse sfaccettature, ha palesato elementi di genere e di classe: gli Ittiti scacciavano una donna finemente vestita, ma non un uomo, laddove per i Greci il pharmakos era di solito un criminale o uno schiavo, oppure apparteneva ai ceti meno abbienti della società. E ancora: tra gli Ittiti era il re ad officiare il rito, mentre fra gli Israeliti il ruolo guida spettava al sommo sacerdote e tra i Greci alla polis nel suo complesso. Infine, tanto il pharmakos quanto il devotus (per i Romani), come pure la figura del "capro espiatorio" (sia tra gli Ittiti sia tra gli Israeliti) riflettono nel rapporto "noi/loro" una separazione e, al contempo, una discriminazione. "Loro", infatti, non sono unicamente gli "altri", ossia i "diversi da noi", ma costituiscono i "nemici": ovvero una categoria necessaria sia nell'intento di individuare (e separare) i tratti identitari degli operatori rituali, sia per procurare (e discriminare) un ostacolo rispetto al quale misurare la tenuta dei valori sociali, politici e religiosi di un sodalizio umano.

Non disponendosi di fonti letterarie concordi sulla morte del pharmakos, possiamo assumere tanto un atteggiamento possibilista quanto uno fortemente critico ${ }^{49}$. La nostra analisi, in ogni caso, oltre ad aver contribuito a chiarire ulteriormente le dinamiche culturali relative all'istituto del pharmakos, ha permesso di ammettere la possibilità che il rito avesse pure altri significati (Geti), oltre a quello, ormai noto, dell'espulsione di una vittima designata, al fine di purificare la comunità investita da una drammatica crisi esistenziale.

Leonardo Sacco

Dipartimento di Scienze giuridiche

Sapienza Università

Piazzale Aldo Moro 5

00185 Roma (Italia)

leonardo.sacco@uniroma1.it

49 Masson 1962, 112 (atteggiamento possibilista); Hughes 1991, 143 (atteggiamento critico). 
Adams Holland 1956

L. Adams Holland, «The Purpose of the Warrior Image of Capestrano», American Journal of Archaeology 60, 3 (1956), 243-274.

BAgLIONI 2013

I. BAGLioni, «Note alla terminologia e al concetto di "mostruoso" nell'antica Grecia", in I. Baglioni (a cura di), Monstra. Costruzione e percezione delle Entità Ibride e Mostruose nel Mediterraneo Antico, Roma 2013, vol. 2, 15-32.

Berggren 1990

K. Berggren, «The Capestrano Warrior and the Numana Head. A Structuralist Semiotic Interpretation», Opuscula Romana 18 (1990), 23-37.

BIANCHI 1970

U. BianchI, «La Storia delle religioni«», in G. Castellani (dir.), Storia delle Religioni, Torino 1970, vol. I, 3-171.

BIANCHI 1989

U. BIANCHI, La religione greca, Torino 1989.

BODNÁr 2000

I. BodnÁr, s.v. Pharmakos, DNP IX, 2000, 750 .

BoËTHIUs 1956

A. Bоётніus, «Livy 8, 10, 12 and the Warrior of Capestrano", Eranos Jahrbuch 54 (1956), 202-210.

BONNECHERE 1994

P. BonneChere, Le sacrifice humain en Grèce ancienne, Athènes-Liège 1994.

BOTTURI 1983

F. BotTuRI, "Strutturalismo e sapere storico», Rivista di Filosofia Neo-Scolastica 75, 4 (1983), 563-581.

BRELICH 1956

A. Brelich, "Appunti su una metodologia», Studi e Materiali di Storia delle Religioni 27 (1956), 1-30.

BRELICH 1966

A. BRELICH, Introduzione alla storia delle religioni, Roma 1966.

\section{BRELICH 1967}

A. Brelich, Presupposti del sacrificio umano (disp. univ.), Roma 1967.

BRELICH 1970-72

A. Brelich, «Perché storicismo e quale storicismo (nei nostri studi)?», Religioni e Civiltà I (1970-1972), 7-28.

\section{BRELICH 1977}

A. Brelich, «La metodologia della Scuola di Roma», in B. Gentili, G. Paioni (a cura di), Il mito greco. Atti del Convegno Internazionale (Urbino, 7-12 maggio 1973), Roma 1977, 3-32.

\section{BREMMER 1983}

J. N. Bremmer, «Scapegoat Rituals in Ancient Greece», Harvard Studies in Classical Philology 87 (1983), 299-320.

BREMMER 2001

J. N. Bremmer, «The Scapegoat between Hittites, Greeks, Israelites and Christians", in R. ALBERTz (Hrsg.), Kult, Konflikt und Versöhnung, München 2001, 174-210.

BRUNAUX 1988

J.-L. Brunaux, The Celtic Gauls: Gods, Rites, and Sanctuaries. Translated by D. Nash, London 1988.

BuCKLEY $2010^{2}$

T. BuCKLEY, Aspects of Greek History 750-323 BC. A Source-Based Approach, London-New York $2010^{2}$.

BURKERT 1972

W. Burkert, Homo necans. Interpretationen altgriechischer Opferriten und Mythen, BerlinNew York 1972.

BURKERT 1979

W. Burkert, Structure and History in Greek Mythology and Ritual, Berkeley 1979.

BURKERT 1985

W. Burkert, Greek Religion, Cambridge [Mass.] 1985.

BuRns 1981

A. Burns, «Athenian Literacy in the Fifth Cen- 
tury B.C.", Journal of the History of Ideas 42, 3 (1981), 371-387.

Carcopino 1935

J. Carcopino, Lostracisme athénien, Paris 1935.

Carmichael 2000

C. Carmichael, «The Origin of the Scapegoat Ritual», Vetus Testamentum 50, 2 (2000), 167 182.

\section{Carter 2003}

J. Carter (edited by), Understanding Religious Sacrifice: A Reader, London-New York 2003.

Casadio 2011

G. Casadio, "Comparative Religion Scholars in Debate: Theology vs History in Letters addressed to Ugo Bianchi», in P. Pachis, D. Wiebe (eds), Chasing Down Religion: In the Sights of the History and the Cognitive Sciences: Essays in Honor of Luther M. Martin, Thessaloniki 2011, 59-80.

Champagne 1992

R. A. Champagne, The Structuralists on Myth. An Introduction, New York 1992.

\section{DAVIES 1977:}

D. DAVIES, "An Interpretation of Sacrifice in Leviticus», Zeitschrift für die alttestamentliche Wissenschaft 89, 3 (1977), 387-398.

de Martino 1953-1954

E. De Martino, «Fenomenologia religiosa e storicismo assoluto", Studi e Materiali di Storia delle Religioni 24-25 (1953-1954), 1-25.

Detienne 1979

M. Detienne (avec J.P. Vernant), La cuisine du sacrifice en pays grec, Paris 1979.

Deubner 1932

L. Deubner, Attische Feste, Berlin 1932.

Deubner 1934

L. Deubner, "Der Pharmakos von Abdera», Studi Italiani di Filologia Classica 11 (1934), 185-192.

Dindorf 1853

G. Dindorf (ed.), Harpocration Lexicon in Decem Oratores Atticos, 2 voll., Oxford 1853.
DURKHeIM I 897

É. Durkheim, Le suicide. Étude de sociologie, Paris 1897.

DURKHeIM 1912

É. Durкheim, Les formes élémentaires de la vie religieuse, Paris 1912.

\section{ELLIS 1881}

R. Ellis, P. Ovidii Nasonis Ibis; ex novis codicibus edidit, scholia vetera commentarium cum prolegomenis, appendice, indice addidit, Oxford 1881.

FARNELL 1907

L. R. Farnell, The Cults of the Greek States, Oxford 1907.

Feinberg 1958

C. L. Feinberg, "The Scapegoat of Leviticus Sixteen", Bibliotheca Sacra 115 (1958), 320333.

FiLORAMO 1997

G. Filoramo, «Comparativismo e Storia delle religioni», Humanitas 52, 4 (1997), 510-527.

FORSDYKE 2005

S. Forsdyke, Exile, Ostracism, and Democracy. The Politics of Expulsion in Ancient Greece, Princeton 2005.

Frazer $1913^{3}$

J. G. Frazer, The Golden Bough, VI: The Scapegoat, London $1913^{3}$.

Gasbarro 1990

N. GASBARRo, "La terza via tracciata da Raffaele Pettazzoni», Studi e Materiali di Storia delle Religioni 56 (1990), 95-199.

GebHaRd 1926

V. Gebhard, Die Pharmakoi in Ionien und die Sybakchoi in Athen, Dissertation, München 1926.

\section{GHenghea 2012}

A. Ghenghea, «Was There a Death Gesture during Early Iron Age? A New Meaning for the 'Warrior from Capestrano'", Buletinul Muzeului Judetean Teleorman 4 (2012), 239-249.

Girard 1972

R. Girard, La violence et le sacré, Paris 1972. 
Girard 1982

R. Girard, Le bouc émissaire, Paris 1982.

Granite 2016

G. Granite, «Understanding the Death and Burial of Northern European Bog Bodies», in C. A. Murray (ed.), Diversity of Sacrifice. Form and Function of Sacrificial Practices in the Ancient World and Beyond, Albany (N.Y.) 2016, 211-222.

\section{Gras 1984}

M. Gras, "Cité grecque et lapidation", in Y. Thomas (éd.), Du châtiment dans la cité. Supplices corporels et peine de mort dans le monde antique. Table ronde de Rome (9-11 novembre 1982), Rome 1984, 75-89.

Green 1998

M. Green, "Humans as Ritual Victims in the Later Prehistory of Western Europe», Oxford Journal of Archaeology 17, 2 (1998), 169-189.

Grottanelli 1999

C. Grottanelli (a cura di), Il sacrificio, RomaBari 1999.

Grottanelli, Parise 1988

C. Grottanelli, N.F. Parise (a cura di), Sacrificio e società nel mondo antico, Roma-Bari 1988.

\section{Gurney 1977}

O. R. Gurney, Some Aspects of Hittite Religion, Oxford 1977.

HARRISON 1903

J. E. Harrison, Prolegomena to the Study of Greek Religion, Cambridge 1903.

Hedley 2011

D. Hedley, Sacrifice Imagined: Violence, Atonement, and the Sacred, New York 2011.

HENRY 1959-1977

R. Henry (éd.), Photius, Bibliothèque, 9 voll., Paris 1959-1977.

Hubert, Mauss 1899

H. Hubert, M. Mauss (eds), Essai sur la nature et la fonction du sacrifice, Paris 1899.

Hughes 1991

D. D. Hughes, Human Sacrifice in Ancient Greece, Abingdon 1991.
KaGAN 1961

D. Kagan, "The Origin and Purpose of Ostracism», Hesperia 30 (1961), 393-401.

KeARNeY 2003

R. Kearney, Strangers, Gods, and Monsters: Interpreting Otherness, London-New Haven 2003.

Kosmin 2015

P. J. Kosmin, «A Phenomenology of Democracy: Ostracism as Political Ritual», Classical Antiquity 34, 1 (2015), 121-162.

LAWSON 1910

J.C. Lawson, Modern Greek Folklore and Ancient Greek Religion. A Study in Survivals, Cambridge 1910 .

LAWTOO 2017

N. Lawtoo, "The Classical World. Sacrifice, Philosophy, and Religion", in J. Alison, W. Palaver (eds), The Palgrave Handbook of Mimetic Theory and Religion, New York 2017, 119-126.

LEACH 1976

E. LeaCH, Culture and Communication: The Logic by Which Symbols Are Connected. An Introduction to the Use of Structuralist Analysis in Social Anthropology, Cambridge 1976.

LEONE 1968

P. A. M. Leone (éd.), Ioannis Tzetzae Historiae, Naples 1968.

LoIsY 1920

A. LoIsy, Essai historique sur le sacrifice, Paris 1920.

MASSON 1962

O. Masson, Les fragments du poète Hipponax, Paris 1962.

MCLean 1990

B. McLean, "On the Revision of Scapegoat Terminology», Numen 37, 2 (1990), 168-173.

McLean 1996

B. H. McLean, The Cursed Christ. Mediterranean Expulsion Rituals and Pauline Soteriology, Sheffield 1996.

Meuli 1975

K. Meuli, Gesammelte Schriften II, Basel 1975. 
Missiou 2011

A. Missiou, Literacy and Democracy in FifthCentury Athens, Cambridge 2011.

Montanari 2001

E. Montanari, Categorie e forme nella Storia delle religioni, Milano 2001.

NAGY 1979

G. NAGY, The Best of the Achaeans, Baltimore 1979.

NiLSSON 1950

M.P. NiLsson, Geschichte der griechischen Religion, München 1950.

Ogden 1997

D. Ogden, The Crooked Kings of Ancient Greece, London 1997.

Palaver 2013

W. Palaver, René Girard's Mimetic Theory (ed. or. Berlin-Münster-Wien-Zürich-London 2011). Translated by Gabriel Borrud, East Lansing 2013.

Paoletti 2004

O. Paoletti, s.v. espulsione, eliminazione, Thesaurus Cultus et Rituum Antiquorum (ThesCRA) - English, French, German, and Italian, ed. V. Lambrinoudakis, J. Ch. Balty, B. Jaeger (Foundation pour le Lexicon Iconographicum Mythologiae Classicae), Los Angeles (Verona) 2004, II, 3.a. Purification/Purification/Reinigung/Purificazione, 33-35.

Parker 2005

R. Parker, Polytheism and Society at Athens, Oxford 2005.

Pestalozza 1930-31

U. Pestalozza, «Le Tharghelie ateniesi», Studi e Materiali di Storia delle Religioni 7 (1930-31), 232-272.

Pettazzoni 1924

R. Pettazzoni, Svolgimento e carattere della Storia delle Religioni. Lezione inangurale pronunciata nell'Università di Roma il 17 gennaio 1924, Bari 1924.

\section{Pettazzoni 1952}

R. Pettazzoni, Italia religiosa, Bari 1952.

\section{Pettazzoni 1959}

R. Pettazzoni, «Il metodo comparativo», $\mathrm{Nu}$ men 6, I (1959), 1-14.

Pfeiffer 1934

R. Pfeiffer, «Zum Papyrus Mediolanensis des Kallimachos», Philologus 89 (1934), 384-385.

PISI 1990

P. PISI, «Storicismo e fenomenologia nel pensiero di Raffaele Pettazzoni», Studi e Materiali di Storia delle Religioni 56 (1990), 246-275.

ReEve 1998

C.D.C. Reeve, Aristotle, Politics, IndianapolisCambridge 1998.

Roussel 1922

P. Roussel, «Le thème du sacrifice volontaire dans la tragédie d'Euripide», Revue Belge de Philologie et d'Histoire 1-2 (1922), 225-240.

SabBatuCCI 1978

D. SabBatucci, Sommario di Storia delle religioni, Roma 1978.

SACCO 2011

L. Sacco, Devotio. Aspetti storico-religiosi di un rito militare romano, Roma 2011.

SACCO 2016

L. SACCO, Lideale di libertà e di tolleranza. Raffaele Pettazzoni (1883-1959) e la coscienza storico-religiosa degli italiani, Roma 2016.

STERN 1991

J. STERN, «Scapegoat Narratives in Herodotus», Hermes 119 (1991), 304-311.

Stoneman 2013

E. Stoneman, «The Available Means of Preservation: Aristotelian Rhetoric, Ostracism, and Political Catharsis», Rhetoric Society Quarterly 43, 2 (2013), 134-154.

TYLOR 1871

E.B. Tylor, Primitive Culture. Researches into the Development of Mythology, Philosophy, Religion, Language, Art and Custom, London 1871.

\section{VAN BAAL 1976}

J. VAN BAaL, «Offering, Sacrifice and Gift», Numen 23, 3 (1976), 161-178. 
Van Gennep 1981

A. VAn Gennep, I riti di passaggio (ed. or. Paris 1909), trad. it. di M.L. Remotti, Torino 1981.

\section{Vernant 1996}

J.-P. Vernant, Entre mythe et politique, Paris 1996.

Vernant, Vidal-Naquet 1977

J.-P. Vernant, P. Vidal-Naquet, Mito e tragedia nell'antica Grecia. La tragedia come fenomeno sociale estetico e psicologico (ed. or. Paris 1972), Torino 1977.

VERSNEL 1977

H. S. Versnel, «Polycrates and His Ring. Two Neglected Aspects», Studi Storico-Religiosi 1 (1977), 37-46.

VERSNEL 1981

H.S. Versnel, «Self-sacrifice, Compensation and the Anonymous Gods", in O. Reverdin, J. Rudhardt (éd.), Le Sacrifice dans l'antiquité
(25-30 Août 1980), Vandœuvres, Genève 1981, 135-194.

WeSTBROOK, LEWIS 2008

R. Westbrook, T. J. Lewis, "Who Led the Scapegoat in Leviticus 16:21?», Journal of Biblical Literature 127, 3 (2008), 417-422.

XeLla 1996

P. Xella, «Il "capro espiatorio" ad Ebla. Sulle origini storiche di un antico rito mediterraneo", Studi e Materiali di Storia delle Religioni 62, 1-2 (1996), 677-684.

\section{YERKES 1952}

R.K. Yerkes, Sacrifice: In Greek and Roman Religions and Early Judaism, New York 1952.

ZATELLI 1998

I. ZATELLI, «The Origin of the Biblical Scapegoat Ritual: The Evidence of Two Eblaite Texts", Vetus Testamentum 48, 2 (1998), 254-263. 